\title{
ENHANCING THE RESOLUTION OF URBAN DIGITAL TERRAIN MODELS USING MOBILE MAPPING SYSTEMS
}

\author{
Y. Feng ${ }^{1 *}$ C. Brenner ${ }^{1}$, M. Sester ${ }^{1}$ \\ ${ }^{1}$ Institute of Cartography and Geoinformatics, Leibniz Universität Hannover, Germany - \\ (yu.feng, claus.brenner, monika.sester)@ikg.uni-hannover.de
}

KEY WORDS: Digital Terrain Models, Mobile Mapping Systems, LiDAR, Overland Flow Modelling

\begin{abstract}
:
Digital Terrain Models (DTMs) are essential surveying products for terrain based analyses, especially for overland flow modelling. Nowadays, many high resolution DTM products are generated by Airborne Laser Scanning (ALS). However, DTMs with even higher resolution are of great interest for a more precise overland flow modelling in urban areas. With the help of mobile mapping techniques, we can obtain much denser measurements of the ground in the vicinity of roads. In this research, a study area in Hannover, Germany was measured by a mobile mapping system. Point clouds from 485 scan strips were aligned and a DTM was extracted. In order to achieve a product with completeness, this mobile mapping produced DTM was then merged and adapted with a DTM product with $0.5 \mathrm{~m}$ resolution from a mapping agency. Systematic evaluations have been conducted with respect to the height accuracy of the DTM products. The results show that the final DTM product achieved a higher resolution $(0.1 \mathrm{~m})$ near the roads while essentially maintaining its height accuracy.
\end{abstract}

\section{INTRODUCTION}

Pluvial floods in urban areas, which are caused by extreme rainfall events, are nowadays a great challenge for many cities. Optimization of the modelling and simulation for such disastrous events are of great interest for both administration and research. Digital Terrain Models (DTMs) provide the primary elevation information. The resolution and accuracy of DTMs plays a crucial role in such simulations. DTMs provided by mapping agencies with a resolution of $2 \mathrm{~m}$ (Mueller et al., 2007) or $1 \mathrm{~m}$ (Garrote and Bras, 1995, Templeton et al., 2014) were often used for overland flow modelling of pluvial floods. However, in these cases, many details of the terrain, which are smaller than the grid size, are not preserved.

Currently, many DTM products with high resolution are generated by Airborne Laser Scanning (ALS). However, the resolution of the final DTM products is limited not only by the scanner accuracy but also the flying height. For cities with dense residential areas and overpasses, occlusions occur and it is hard to achieve very dense measurements on the ground using ALS. UAV (Unmanned Aerial Vehicle) is another popular platform for generating high resolution DTMs, however, it suffers similar issues as ALS. Due to the perspective from above, trees, huge buildings may lead to insufficient measurements on the ground. Therefore, mobile mapping, as an efficient surveying tool on the ground, can be used as a supplementary source to improve the resolution of DTMs in urban areas. Nowadays, mobile mapping technologies are widely applied for building 3D city models and mapping road infrastructures (Yang et al., 2013, Guan et al., 2014, Yang et al., 2017). Only in the recent ten years, high resolution DTMs with sub metric grids were generated by mobile mapping systems and utilized for urban inundation studies (Fewtrell et al., 2011, Sampson et al., 2012, Ozdemir et al., 2013). In their research, a DTM with $10 \mathrm{~cm}$ resolution in an area covering four streets $\left(0.11 \mathrm{~km}^{2}\right)$ was generated. The DTM and its re-sampled DTM in $0.25 \mathrm{~m}$,

\footnotetext{
${ }^{*}$ Corresponding author
}

$0.5 \mathrm{~m}, 1 \mathrm{~m}, 2 \mathrm{~m}$ and $5 \mathrm{~m}$ were used in the overland flow modelling. The results show that higher resolution urban inundation analyses are beneficial for the simulation (Sampson et al., 2012). However, in their study, only the DTM in the area of the roads was used. This leads to an inconsistency with respect to the complete area. Another study was conducted with the mobile mapping measurements along two streets $\left(0.16 \mathrm{~km}^{2}\right)$ in London, the United Kingdom (Leitão et al., 2016). Merging algorithms were provided to achieve a product with better completeness.

In both studies above, only very small study areas with a few streets were investigated. However, for a surveying in a relative larger area, it is inevitable that locations (such as intersections) may be passed by the mobile mapping system several times. Due to GNSS (Global Navigation Satellite System) errors, especially multi-path effects, multiple scan strips of larger urban areas may suffer significant loop closure problems, which lead to inconsistent height and position values. For generating a higher resolution DTM product of extended areas, the consistent alignment of point clouds from multiple scan strips is a great challenge. Also, since the relative alignment of scan strips increases the precision only, but not the absolute accuracy, one is interested in an additional alignment to an absolute reference, such as a given DTM, especially with respect to the height.

In this research, we are aiming to provide a better DTM product with good completeness and higher resolution by merging the mobile mapping data with a given DTM. Our DTM product can be used by hydrologists in order to analyze how high resolution DTMs improve their hydraulic simulations, in contrast to stateof-the-art simulation approaches using coarse DTM data. Further algorithms can also be developed based on this higher resolution DTM product for road surface monitoring and road cracks detection. In the following section, we introduce the mobile mapping system and the reference DTM used in this research. Our pipeline method is described in Section 3 with three major steps: alignment, ground filtering and merging of DTMs of different resolution. Afterwards, a comparison is conducted not only between our DTM and the reference DTM, but also using leveling 
measurements of manholes in this area. At the end, we conclude and discuss possible future work.

\section{DATA}

\subsection{Mobile Mapping System for Data Acquisition}

In this research, a Riegl VMX-250 mobile mapping system (shown in Figure 1) was used for data acquisition in HannoverRicklingen, Germany. This system contains two Riegl VQ-250 laser scanners, which can measure 600,000 points per second (Riegl, 2012). The measurement range is limited to 200 meters to either side of the vehicle and the scanner accuracy is given as $10 \mathrm{~mm}$.

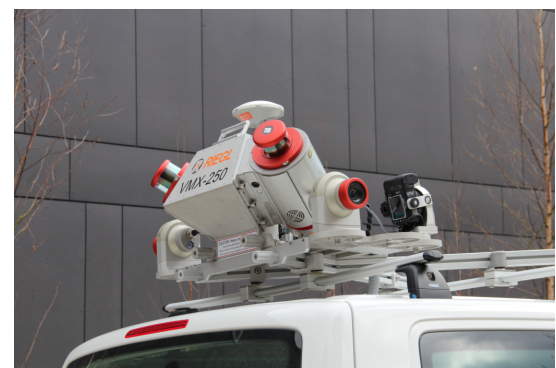

Figure 1. Mobile mapping system - Riegl VMX-250

Positions and orientations of this system are provided by a GNSS receiver, an Inertial Measurement Unit (IMU) and an external Distance Measurement Instrument (DMI), attached to one wheel of the vehicle. In post-processing, positions were corrected according to reference data provided by the SAPOS service (LGLN, 2017) in Hannover, Germany. The corrected trajectories can achieve an accuracy about $0.1-0.3 \mathrm{~m}$ in height and $0.2 \mathrm{~m}$ in position in urban areas (Hofmann and Brenner, 2016, Schachtschneider et al., 2017). We carefully planned the measurement campaign and captured almost all the accessible paths in the study area (shown in Figure 2). In order to avoid redundant data acquisition at the same place when the vehicle stops, we pause the data acquisition shortly and restart it when the vehicle begins to move. Therefore, in total, 485 scan strips were acquired by our measurement campaign in the study area Hannover-Ricklingen.

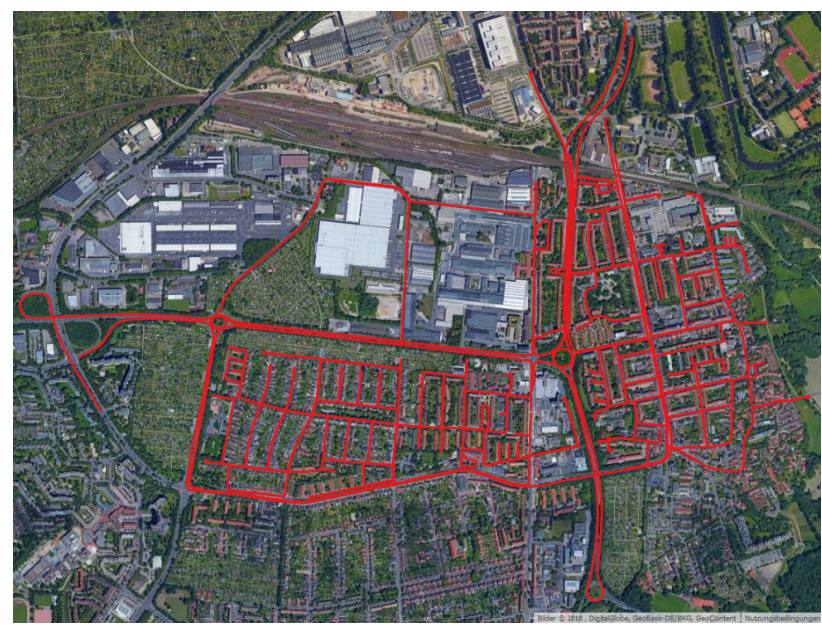

Figure 2. Trajectories of measurement campaign in the study area Hannover-Ricklingen (Basemap: Google Map)

\subsection{Reference DTM from Mapping Agencies}

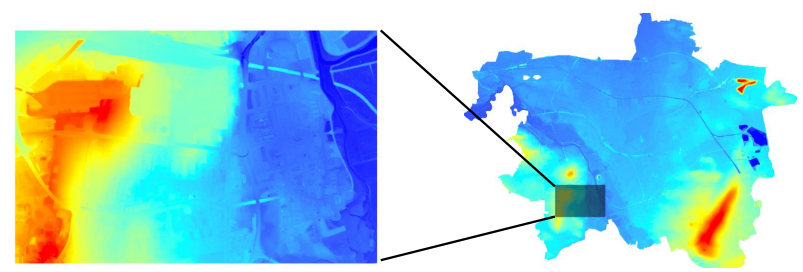

Figure 3. DTM of Hannover-Ricklingen (left) and Hannover (right) in $0.5 \mathrm{~m}$ resolution

A DTM product from the mapping agency of the federal state Lower Saxony, Germany (LGLN, Landesamt für Geoinformation und Landesvermessung Niedersachsen) in $0.5 \mathrm{~m}$ resolution was used as a reference DTM in this research (as shown in Figure 3). This product was generated from airborne laser scans acquired in 2009. It covers our whole study area in Hannover-Ricklingen $\left(3,201 \times 2,045 \mathrm{~m}^{2}\right)$. The documentation states that this product has an accuracy of $\pm 0.2 \mathrm{~m}$ in height and $\pm 0.3 \mathrm{~m}$ in position (LGLN, 2018). A point cloud was generated based on this product, which is used for further experiments.

\section{METHODS}

Many researchers have focused on different stages of producing a high resolution DTM. The pipeline of stages we use is summarized in Figure 4. Scan strips were firstly aligned and saved in $15 \times 15 \mathrm{~m}^{2}$ tiles. Subsequently, ground filtering was applied for each single tile to extract bare-earth. Since the mobile mapping system can only measure a range of about $200 \mathrm{~m}$ around the roads, a merging step is necessary to achieve completeness of the data. Thus, a survey product from the mapping agency with lower resolution can be used to fill the regions inaccessible to mobile mapping. In order to achieve a smooth transition at the connecting borders of DTMs in different resolution, interpolation was applied in a buffer area around the mobile mapping DTM to adapt these height changes. All tiles are assembled to build the final DTM product. The output of our approach is a point cloud, which can either be used directly for generating a TIN (Triangulated Irregular Network) or to be interpolated to a higher resolution raster than the reference DTM. Three essential steps can be identified, which are: $(i)$ alignment of laser scans from different epochs, (ii) ground filtering, and (iii) merging of DTMs with different resolution. They are explained in the following sections.

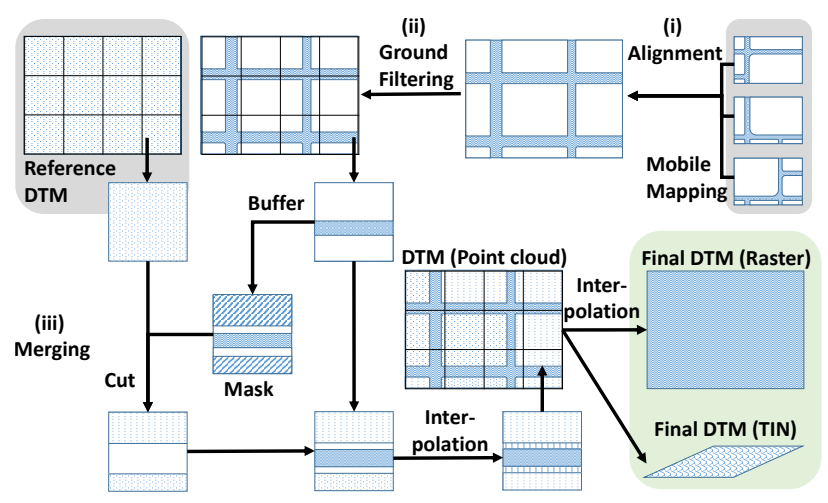

Figure 4. Work flow of our pipeline method 


\subsection{Alignment of Laser Scans}

The alignment of laser scans can be achieved by a rigid body transformation, requiring to estimate rotation and translation parameters. For this, corresponding point pairs need to be identified, which can be based on the proximity of points in the original point cloud. Alternatively, feature points can be extracted from 2D range images (Steder et al., 2011, Feng et al., 2016) or 3D point clouds (Zhong, 2009, Rusu et al., 2009), which are subsequently assigned based on their descriptors. In the former case, since the correctness of point pair assignments cannot be assessed, they are usually repeated after each estimation of the transformation, leading to the well-known iterative closest point (ICP) algorithm (Besl and McKay, 1992). In the latter case, a non-iterative procedure may be obtained, however a sufficient number of unique feature point pairs must be present in the scene.

In case of mobile mapping, the required transformation varies along the scan strips (which usually have a length of several hundred meters). Thus, a rigid body transformation cannot be used. Instead, a rigorous error modelling leads to a function which corrects the exterior orientation of the sensor platform along the trajectory, and which is estimated using the residuals between the scanned points of each strip, a procedure known as strip adjustment. In addition, since some parts of the scene may be covered by a large number of scan strips, pairwise correspondences will not scale well, since they lead to a quadratic number of point correspondences. Therefore, all scans are iteratively aligned to a temporary object known as latent map (Huang and Anguelov, 2010). The overall procedure is similar to a photogrammetric bundle adjustment using tie points, with the exception that the entire surface of the project area is used as a tie surface. It is solved using alternate least squares adjustment as follows:

1. Each scan strip is preprocessed individually, obtaining local surface normal vectors and a segmentation into continuous regions,

2. all scan strips are used to estimate a global, latent map,

3. residuals between points and this latent map are used to derive observation equations (which relate the latent map surface with the corrections of the exterior orientations along the scan strip),

4. additional equations are introduced which enforce the GNSS/IMU observed positions and orientations, as well as the continuity of the sensor system trajectory,

5. a global adjustment of positions and orientations is carried out,

6. the corrected sensor orientations are used to correct all point clouds, and the procedure is iterated, starting from (2), until convergence.

For the Ricklingen project area, the original scan contained 5.1 billion points in 485 scan strips, from which 3.6 billion remained after segmentation and were used in the strip adjustment. 1.9 billion surface elements (of size $2 \times 2 \mathrm{~cm}^{2}$ ) and 781,000 exterior orientation correction parameters were estimated. After adjustment, the standard deviation of the surface elements is close to the laser scanner precision (shown in Figure 5). The approach is described in more detail in (Brenner, 2016).

We compared the DTMs generated from this data with and without the alignment step (shown in Figure 6). Significant improvements have been achieved which increase the smoothness of the DTM surface.

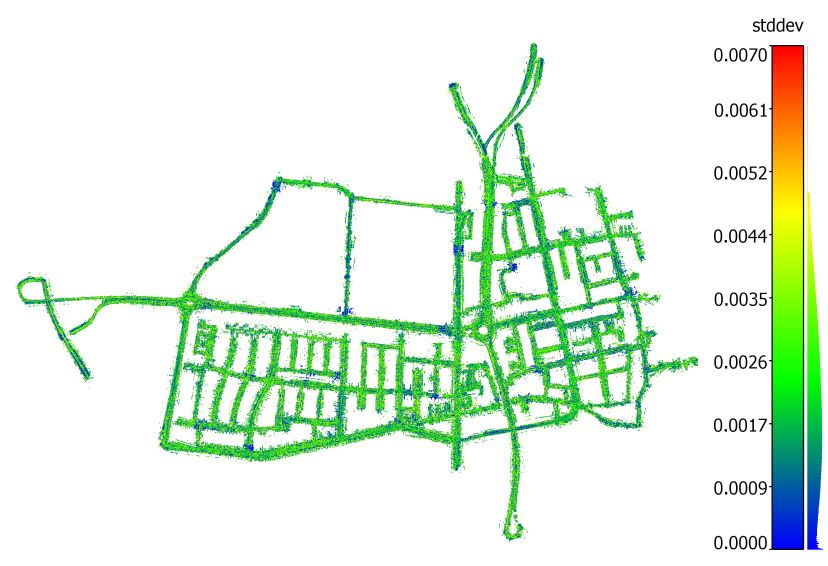

Figure 5. Precision Map (temperature scale, blue for $0 \mathrm{~mm}$ and red for $7 \mathrm{~mm}$ )
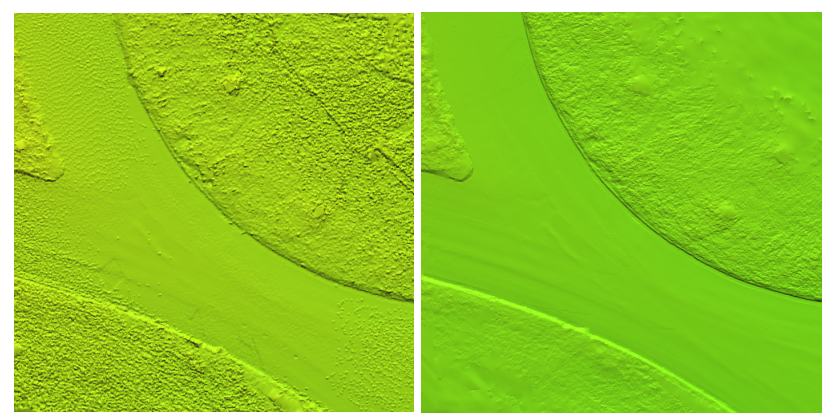

Figure 6. Comparison of DTMs generated without alignment (left) and with alignment (right)

\subsection{Ground Filtering}

Currently, most of the research about DTM extraction is based on ALS data and many algorithms have been proposed in the last 30 years. The performance of eight different methods was compared based on a benchmark data set during a contest held by ISPRS (International Society for Photogrammetry and Remote Sensing) (Vosselman and Maas, 2010, Sithole and Vosselman, 2004). Among these approaches, methods from Axelsson (Axelsson, 1999) and Wack (Wack and Wimmer, 2002) have achieved a relatively good performance for this task. In recent years, ground filtering can also be achieved through $3 \mathrm{D}$ point cloud classification, e.g. using deep convolutional neural networks for DTM extraction (Hu and Yuan, 2016). For filtering mobile mapping point clouds, previous researchers have used the commercial software TerraScan (Vaaja et al., 2011), which was originally designed for airborne laser scans. The algorithm of Axelsson (Axelsson, 1999) was realized in this software for DTM extraction (Brovelli and Lucca, 2011).

Since improving the ground filtering algorithms is not the focus of this study, in our research, we used the high accuracy reference DTM as prior information and implemented one method from literature (Wack and Wimmer, 2002) to extract terrain from mobile mapping point clouds. We first calculated the maximum height $H_{\text {max }}$ and minimum height $H_{\text {min }}$ of the reference DTM for each tile. A threshold $\epsilon$ was set as $0.5 \mathrm{~m}$ and mobile mapping measurements with a height outside the range $\left[H_{\min }-\epsilon, H_{\max }+\epsilon\right]$ were removed for each tile. In this way, only the points near or connected to the terrain are preserved. Then, the method proposed by Wack (Wack and Wimmer, 2002) was realized. As reported in the comparative study, this method performs well for low objects and 
low vegetation (Sithole and Vosselman, 2004). It uses a hierarchical framework. It begins with generating a $9 \mathrm{~m}$ resolution raster DTM from the raw ALS point cloud. The height value of each cell is estimated according to the lowest data point height from 99 percentile of all data points within the raster element. With the height value in each grid, a maximum allowed height deviation can help to roughly exclude the large buildings and dense vegetation. In the next step, a Laplacian of Gaussian (LoG) operation was applied for detecting non-terrain objects. Subsequently, a $3 \mathrm{~m}$ raster can be estimated with the same procedure, followed by $1 \mathrm{~m}, 0.33 \mathrm{~m}$ and $0.11 \mathrm{~m}$ grids. In our case, since most of the points from large buildings and trees are already removed by the prior height information from the reference DTM, instead of using a $9 \mathrm{~m}$ grid size (as in the original work) as starting point, a smaller grid size of $1 \mathrm{~m}$ was used for filtering mobile mapping measurements. Subsequently, the same procedure was applied for the grid sizes $0.33 \mathrm{~m}$ and $0.11 \mathrm{~m}$. In Figure 7, we compare a tile of the point cloud before and after ground filtering.
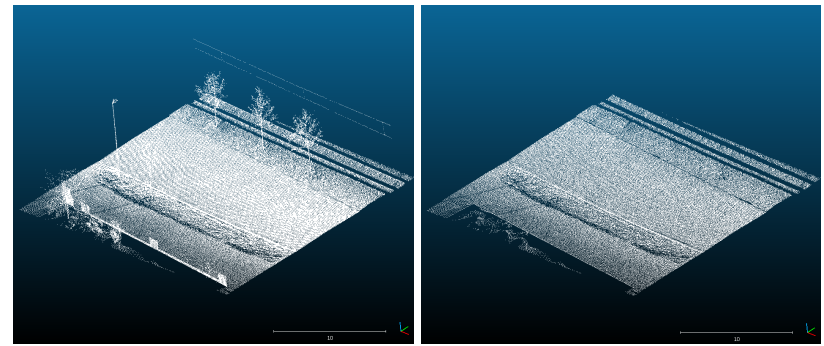

Figure 7. Comparison of point cloud before ground filtering (left) and after ground filtering (right)

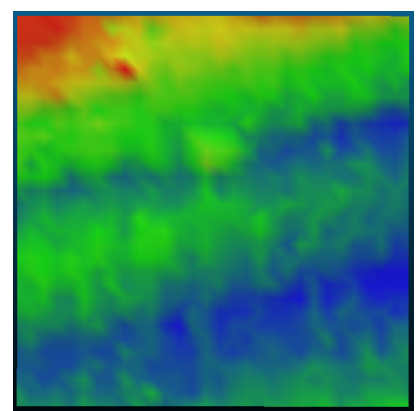

(a) DTM from mapping agency in lower resolution

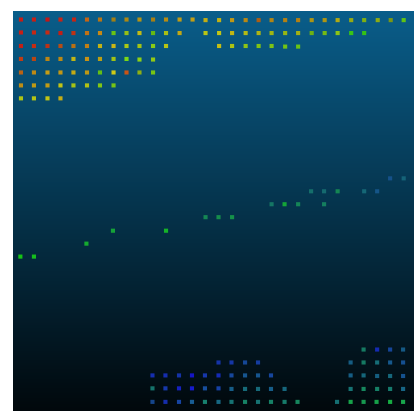

(c) Adapted DTM filling

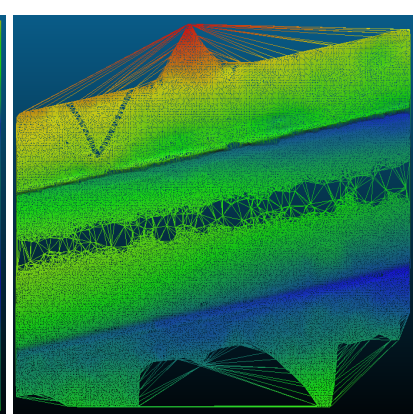

(b) Measurements after ground filtering

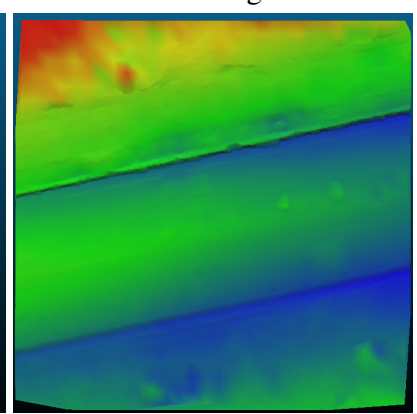

(d) Merged DTM
Figure 8. Merging of DTMs with differing resolution

\subsection{Merging of DTMs}

Directly superimposing DTMs from different sensors and with different resolution may lead to significant height inconsistencies at their connecting borders. Therefore, merging is neces- sary for building a product with good consistency and completeness. Conventional Geographic Information Systems (GIS) software provides standard methods for raster mosaics, e.g. COVER, MEAN and BLEND (Leitão et al., 2016, ESRI, 2018). For the overlapping area, the COVER method chooses the values from the top DTM, in other words, it superimposes the DTMs. The MEAN method assigns the average height values of two DTMs for the overlapping area, while the BLEND method uses the weighted average. However, the COVER method may lead to significant height jumps at connecting borders of the DTMs and the other two methods may not preserve the local height accuracy of the high resolution DTM.

For merging DTMs with different resolution, MBlend (Modified Blend) was proposed in order to preserve the accuracy of the most accurate DTM, and it achieved a better performance in comparison to the above mentioned methods (Leitão et al., 2016). The height differences of points on the boundary of the high resolution DTM are estimated. Only the lower resolution DTM is updated and the height difference is propagated to the whole extent of the lower resolution DTM through interpolation. In our research, a similar idea as MBlend was used to merge the high resolution DTM point cloud into the DTM point cloud in lower resolution. The only difference is that we extracted a predefined buffer area for the high resolution DTM and linear interpolation was only conducted in that area to adapt height changes at the borders. This preserves the height accuracy of the lower resolution DTM and limits the updates for the lower resolution DTM to a certain area. As shown in Figure 8, the lower resolution DTM (Figure 8a) was firstly clipped based on the mobile mapping measurements (Figure $8 \mathrm{~b}$ ). Height differences at the connecting borders were estimated and the DTM filling (Figure 8c) was adapted. Together with the mobile mapping measurements, a merged DTM (Figure 8d) was generated. This point cloud has a high point density in the vicinity of roads and a lower point density for rest of the study area.

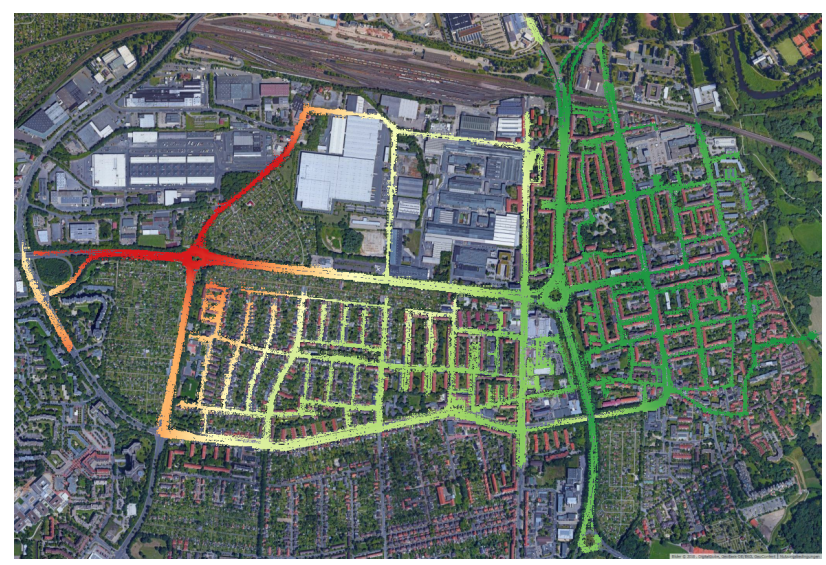

Figure 9. DTM generated from aligned mobile mapping point cloud (Basemap: Google Maps)

\section{RESULTS AND DISCUSSION}

\subsection{Results}

In our study area in Hannover-Ricklingen, 485 scan strips were aligned and partitioned into $15 \times 15 \mathrm{~m}^{2}$ tiles. A DTM (shown in Figure 9) was generated after ground filtering of each tile, where the color indicates the height. Almost all of the accessible space 


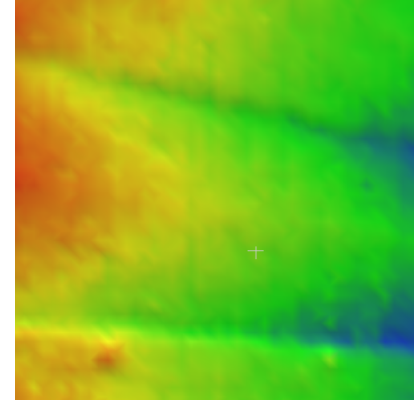

(a) Road - Before

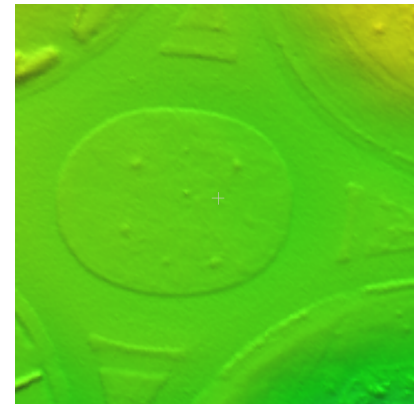

(c) Roundabout - Before

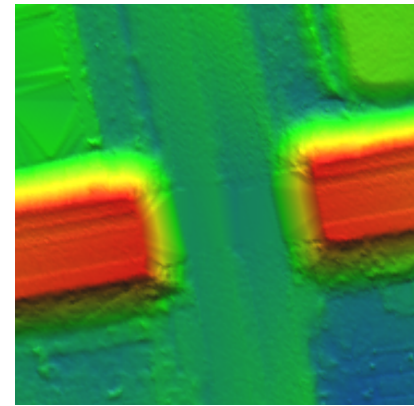

(e) Bridge - Before

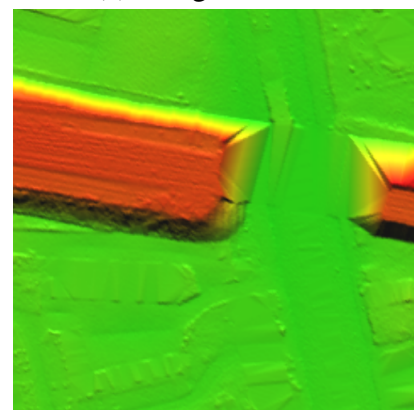

(g) Correction - Before

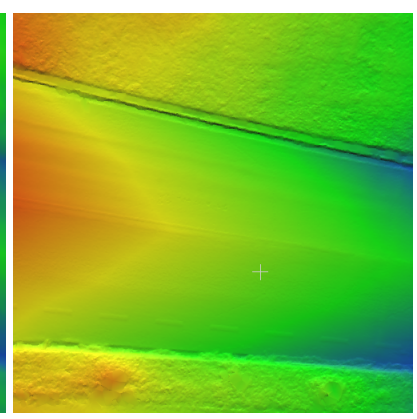

(b) Road - After

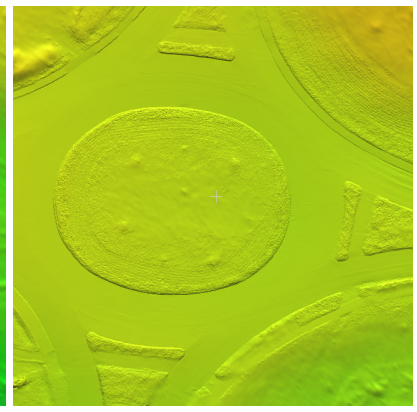

(d) Roundabout - After

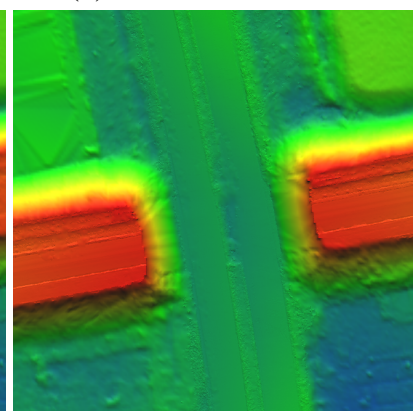

(f) Bridge - After

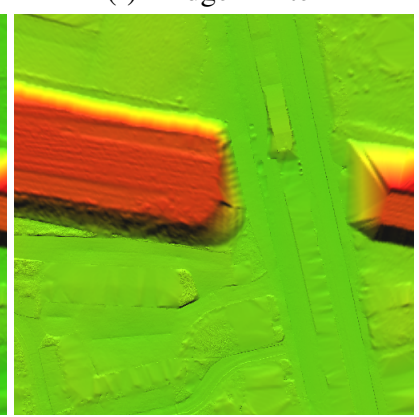

(h) Correction - After
Figure 10. Comparison of DTMs before and after updates in the scenarios: road, bridge, roundabout and correction for interpolation errors

in this area was acquired with high density ground measurements using the mobile mapping system. The dense mobile mapping point cloud was then resampled with a distance of $0.1 \mathrm{~m}$. At the end, a DTM point cloud which merges the mobile mapping measurements and the DTM point cloud ( $0.5 \mathrm{~m}$ resolution) from the mapping agency was generated.

This updated higher resolution DTM is then compared with the reference DTM from the mapping agency with lower resolution (shown in Figure 10). Figure 10a and Figure 10b show the road area mapped by the mobile mapping system, where the whole area has been updated with dense measurements. Curb stones are preserved with proper height difference. For a larger area, a roundabout is compared in Figure 10c and 10d. Apart from the well preserved curb stones, the updated DTM shows better contour, and height differences of the pedestrian passes are clearer. At the four corners of this screen shot, the DTM with lower resolution is merged and no significant height jumps can be observed. In Figure 10e and 10f, even though both roads on the bridge and under the bridge were measured, only the proper parts on the roads were updated. An ALS generated DTM may also contain interpolation errors because of obstacles, such as bridges. Such errors can be corrected, when the roads under the bridge were measured by the mobile mapping system (Figures 10g, 10h).

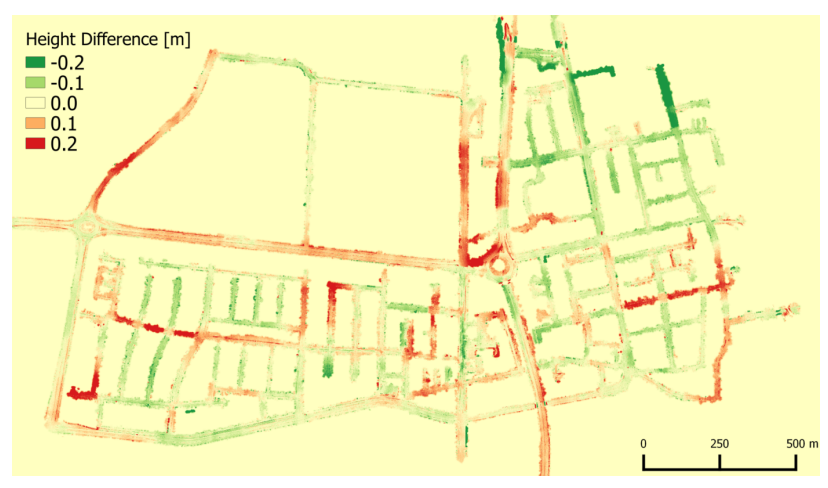

Figure 11. Difference between mobile mapping DTM and reference DTM

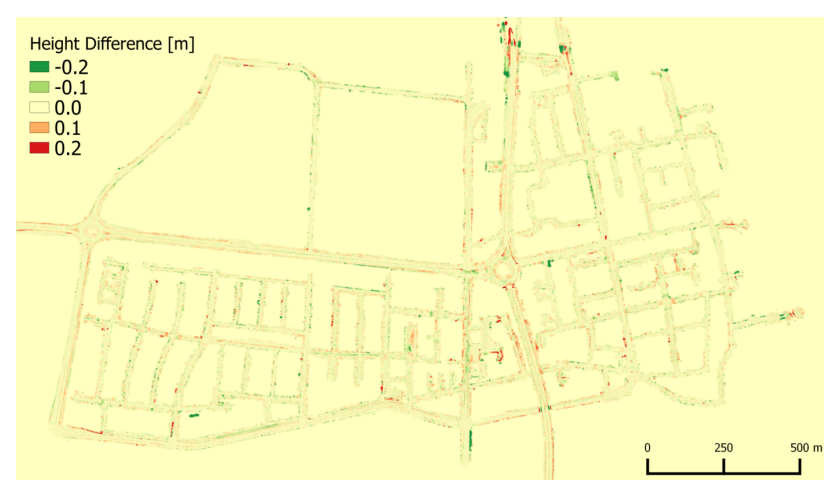

Figure 12. Difference between mobile mapping DTM+ and reference DTM

\begin{tabular}{|l|c|c|}
\hline & Mean [m] & Standard Deviation [m] \\
\hline Mobile mapping DTM & 0.0020 & 0.0989 \\
Mobile mapping DTM+ & -0.0022 & 0.0674 \\
\hline
\end{tabular}

Table 1. Height difference between mobile mapping generated DTMs and reference DTM

\subsection{Evaluation and Discussion}

Subsequently, the height accuracy of the final mobile mapping DTM was analyzed. Since this product does not preserve a good absolute height accuracy, further adaptions have been made to generate a mobile mapping DTM+, which can achieve a high resolution and preserve the height accuracy at the same time.

In order to achieve the best match between the mobile mapping DTM and reference DTM, we calculated a map of height differences. Since many places have no enhancements by using mobile 
mapping systems, we excluded the unchanged pixels and calculated the mean and standard deviation of the height differences (Table 1). The spatial distribution of the height differences is shown in Figure 11. Significant deviations can be observed at the end of scan strips. In order to understand whether the mobile mapping measurements can improve the height accuracy of the DTM, 1,405 leveling measurements of the manholes in this region were used for a height accuracy evaluation. These measurements were firstly compared with the reference DTM. We rejected the points with a height difference larger than $0.15 \mathrm{~m}$ as outliers. Comparisons are given based on this subset of leveling measurements. The height differences are calculated (as summarized in Table 2). The spatial distribution of the difference between leveling measurements and reference DTM is shown in Figure 13a, while the comparison to the mobile mapping DTM is shown in $13 b$.

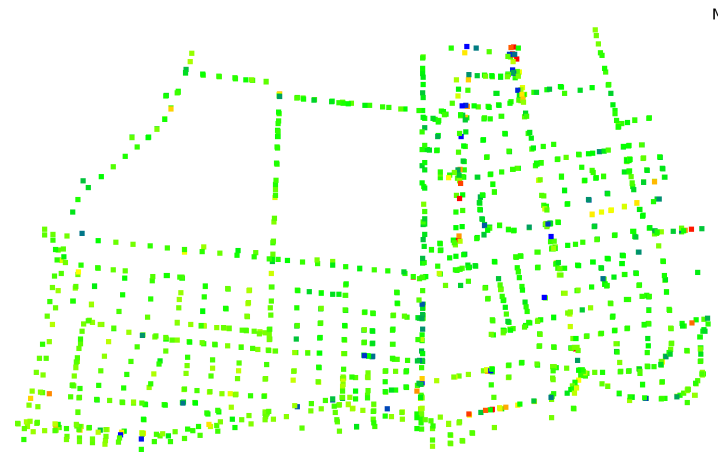

(a) Reference DTM

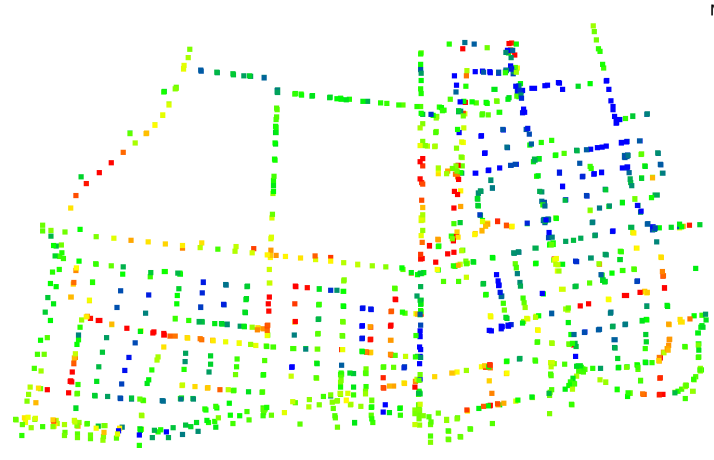

(b) Mobile mapping DTM

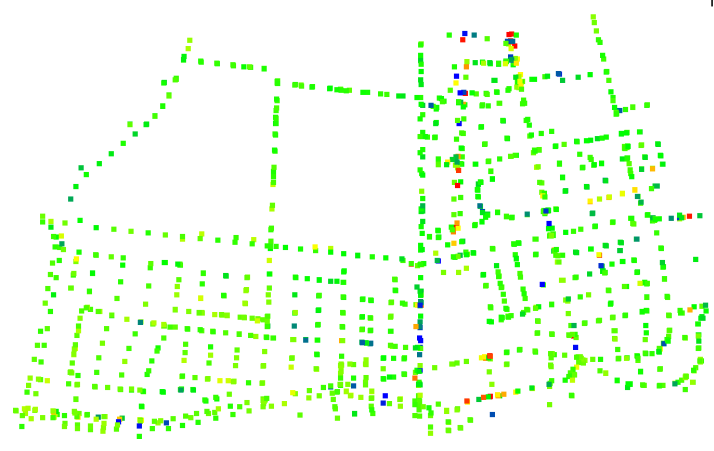

(c) Mobile mapping DTM+

Figure 13. Height Difference between Leveling Measurements of manholes and the DTMs (red for $0.12 \mathrm{~m}$ and blue for $-0.12 \mathrm{~m}$ )

From the results above, we can identify that the reference DTM generated from ALS has a higher absolute height accuracy than the DTM generated by mobile mapping measurements. Therefore, before the merging step, the height differences between the

\begin{tabular}{|l|c|c|}
\hline & Mean [m] & Standard Deviation [m] \\
\hline Reference DTM & -0.0202 & 0.0307 \\
Mobile mapping DTM & -0.0197 & 0.0837 \\
Mobile mapping DTM+ & -0.0193 & 0.0328 \\
\hline
\end{tabular}

Table 2. Height difference between leveling measurements of manholes and the DTMs

mobile mapping DTM and reference DTM were calculated. We rejected the outliers and calculated the height updates for each tile of mobile mapping measurements in $10 \mathrm{~cm}$ resolution using linear interpolation. In this way, we adjusted the height of the mobile mapping measurements to match the reference DTM. We name this product mobile mapping DTM+. Subsequently, this DTM was compared to the reference DTM (shown in Figure 12). This shows that the adjustment was able to remove large parts of the height deviation. The standard deviation of the height differences is also much smaller than the DTM without adaption (summarized in Table 1).

In comparison to manhole leveling measurements, this product achieved a similar standard deviation as the reference DTM (summarized in Table 2) which preserves the good absolute height accuracy. From our results, it becomes clear that while the scan strip alignment is able to increase the precision considerably, the resulting model may still include substantial absolute height deviations, which should be removed by introducing additional reference information. After the adaption we applied, we improved the resolution of the DTM in the vicinity of roads without a noticeable loss of height accuracy. Figure 14 shows the effect of height adjustment. On the left, no height adjustment is used, and the blending of both height models introduces artefacts (nonexistent terrain structures) along the buffer areas. These are removed after adjustment (on the right).
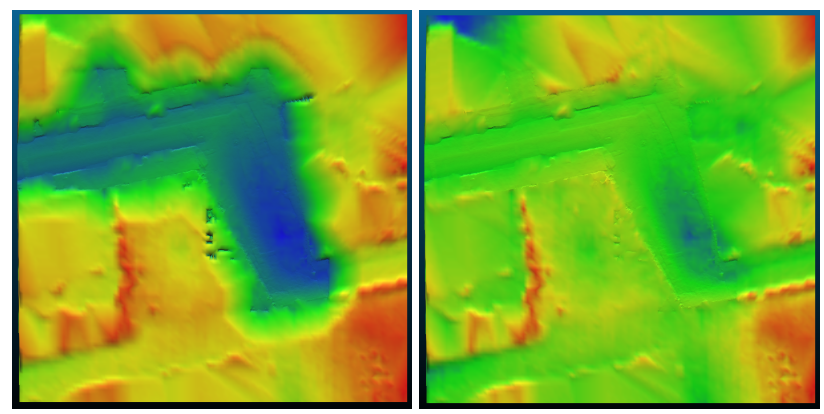

Figure 14. Comparison of DTMs before (left) and after (right) adjustment of the height

\section{CONCLUSIONS}

In this paper, we proposed a pipeline of methods to enhance an urban DTM to a higher resolution using mobile mapping systems. Registration of the point clouds from different epochs played an important role for producing such a product with high precision. Bare ground surface was extracted based on a well-defined hierarchical approach. A merging method considering height differences at the connecting borders of two DTMs was used to improve the consistency and completeness of the DTM product. Through the processing steps above, a very precise and accurate DTM was derived in the vicinity of the roads. This can be the basis for a more detailed overland flow modelling in urban areas. At the same time, it may be used to correct interpolation errors in the reference DTM products from mapping agencies. 
Even though the currently used ground filtering algorithm performs well, a more state-of-the-art method should be applied for even better performance. Occlusions often lead to missing ground measurements, especially because of parked vehicles. Breaklines should be integrated in the next step to achieve a better interpolation in areas where measurements are missing. Previous studies (Sampson et al., 2012, Leitão et al., 2016) have proved the benefit of higher resolution DTMs for overland flow modelling, however, only in a range of several streets. Within our project (Fuchs et al., 2017), one of the next steps will be to investigate the benefit of such high resolution models for the purpose of larger scale overland flow modelling.

\section{ACKNOWLEDGEMENTS}

The authors would like to acknowledge the support from the BMBF funded research project "EVUS - Real-Time Prediction of Pluvial Floods and Induced Water Contamination in Urban Areas" (BMBF, 03G0846A). We also gratefully acknowledge the support of NVIDIA Corporation with the donation of a GeForce Titan X GPU used for this research.

\section{REFERENCES}

Axelsson, P., 1999. Processing of laser scanner data - algorithms and applications. ISPRS Journal of Photogrammetry and Remote Sensing 54(2-3), pp. 138-147.

Besl, P. J. and McKay, N. D., 1992. A method for registration of 3-D shapes. IEEE Transactions on Pattern Analysis and Machine Intelligence 14(2), pp. 239-256.

Brenner, C., 2016. Scalable estimation of precision maps in a mapreduce framework. In: Proceedings of the 24th ACM SIGSPATIAL International Conference on Advances in Geographic Information Systems, GIS '16, ACM, New York, NY, USA, pp. 27:1-27:10.

Brovelli, M. A. and Lucca, S., 2011. Filtering LiDAR with GRASS: overview of the method and comparisons with terrascan. Ital. J. Remote Sens.-Rivista Italiana Di Telerilevamento 43(2), pp. 93-105.

ESRI, 2018. Mosaic Methods. http://pro.arcgis.com/en/ pro-app/tool-reference/data-management/mosaic.htm (30 March 2018)

Feng, Y., Schlichting, A. and Brenner, C., 2016. 3D feature point extraction from LiDAR data using a neural network. ISPRS International Archives of the Photogrammetry, Remote Sensing and Spatial Information Sciences XLI-B1, pp. 563-569.

Fewtrell, T. J., Duncan, A., Sampson, C. C., Neal, J. C. and Bates, P. D., 2011. Benchmarking urban flood models of varying complexity and scale using high resolution terrestrial LiDAR data. Physics and Chemistry of the Earth, Parts A/B/C 36(7-8), pp. 281-291.

Fuchs, L., Graf, T., Haberlandt, U., Kreibich, H., Neuweiler, I., Sester, M., Berkhahn, S., Feng, Y., Peche, A., Rözer, V., Sämann, R., Shehu, B. and Wahl, J., 2017. Real-time prediction of pluvial floods and induced water contamination in urban areas. In: 14th IWA/IAHR International Conference on Urban Drainage, pp. 620-628.

Garrote, L. and Bras, R. L., 1995. A distributed model for realtime flood forecasting using digital elevation models. Journal of Hydrology 167(1-4), pp. 279-306.
Guan, H., Li, J., Yu, Y., Wang, C., Chapman, M. and Yang, B., 2014. Using mobile laser scanning data for automated extraction of road markings. ISPRS Journal of Photogrammetry and Remote Sensing 87, pp. 93-107.

Hofmann, S. and Brenner, C., 2016. Accuracy assessment of mobile mapping point clouds using the existing environment as terrestrial reference. ISPRS - International Archives of the Photogrammetry, Remote Sensing and Spatial Information Sciences XLI-B1, pp. 601-608.

Hu, X. and Yuan, Y., 2016. Deep-learning-based classification for DTM extraction from ALS point cloud. Remote Sensing 8(9), pp. 730.

Huang, Q. X. and Anguelov, D., 2010. High quality pose estimation by aligning multiple scans to a latent map. In: 2010 IEEE International Conference on Robotics and Automation, pp. 13531360 .

Leitão, J. P., Prodanović, D. and Maksimović, Č., 2016. Improving merge methods for grid-based digital elevation models. Computers \& Geosciences 88, pp. 115-131.

LGLN, 2017. SAPOS-Satellitenpositionierungsdienst - Landesamt für Geoinformation und Landesvermessung Niedersachsen. https://www.lgln.niedersachsen.de/download/ 121562/Produktinformation_SAPOS .pdf (30 March 2018).

LGLN, 2018. Digitale Geländemodelle (DGM) - Landesamt für Geoinformation und Landesvermessung Niedersachsen. https://www.lgln.niedersachsen.de/download/ 122447/Produktinformation/_DGM.pdf (30 March 2018).

Mueller, E. N., Wainwright, J. and Parsons, A. J., 2007. Impact of connectivity on the modeling of overland flow within semiarid shrubland environments. Water Resources Research 43, pp. 9.

Ozdemir, H., Sampson, C., de Almeida, G. A. and Bates, P., 2013. Evaluating scale and roughness effects in urban flood modelling using terrestrial LiDAR data. Hydrology and Earth System Sciences 10 , pp. 5903-5942.

Rusu, R. B., Blodow, N. and Beetz, M., 2009. Fast point feature histograms (FPFH) for 3D registration. In: Robotics and Automation, 2009. ICRA'09. IEEE International Conference on, IEEE, pp. 3212-3217.

Sampson, C. C., Fewtrell, T. J., Duncan, A., Shaad, K., Horritt, M. S. and Bates, P. D., 2012. Use of terrestrial laser scanning data to drive decimetric resolution urban inundation models. Advances in water resources 41 , pp. 1-17.

Schachtschneider, J., Schlichting, A. and Brenner, C., 2017. Assessing temporal behavior in lidar point clouds of urban environments. The International Archives of Photogrammetry, Remote Sensing and Spatial Information Sciences 42, pp. 543.

Sithole, G. and Vosselman, G., 2004. Experimental comparison of filter algorithms for bare-earth extraction from airborne laser scanning point clouds. ISPRS Journal of Photogrammetry and Remote Sensing 59(1-2), pp. 85-101.

Steder, B., Rusu, R. B., Konolige, K. and Burgard, W., 2011. Point feature extraction on $3 \mathrm{D}$ range scans taking into account object boundaries. In: Robotics and automation (icra), 2011 ieee international conference on, IEEE, pp. 2601-2608.

Templeton, R. C., Vivoni, E. R., Méndez-Barroso, L. A., Pierini, N. A., Anderson, C. A., Rango, A., Laliberte, A. S. and Scott, R. L., 2014. High-resolution characterization of a semiarid watershed: Implications on evapotranspiration estimates. Journal of hydrology 509, pp. 306-319. 
Vaaja, M., Hyyppä, J., Kukko, A., Kaartinen, H., Hyyppä, H. and Alho, P., 2011. Mapping topography changes and elevation accuracies using a mobile laser scanner. Remote Sensing 3(3), pp. 587-600.

Vosselman, G. and Maas, H.-G., 2010. Airborne and terrestrial laser scanning. CRC Press, pp. 135-167.

Wack, R. and Wimmer, A., 2002. Digital terrain models from airborne laserscanner data-a grid based approach. International Archives of Photogrammetry Remote Sensing and Spatial Information Sciences 34(3/B), pp. 293-296.

Yang, B., Dong, Z., Liu, Y., Liang, F. and Wang, Y., 2017. Computing multiple aggregation levels and contextual features for road facilities recognition using mobile laser scanning data. ISPRS Journal of Photogrammetry and Remote Sensing 126, pp. 180-194.

Yang, B., Fang, L. and Li, J., 2013. Semi-automated extraction and delineation of 3D roads of street scene from mobile laser scanning point clouds. ISPRS Journal of Photogrammetry and Remote Sensing 79, pp. 80-93.

Zhong, Y., 2009. Intrinsic shape signatures: A shape descriptor for 3D object recognition. In: Computer Vision Workshops (ICCV Workshops), 2009 IEEE 12th International Conference on, IEEE, pp. 689-696. 